\title{
Association between Testicular Microlithiasis, Testicular Cancer, Cryptorchidism and History of Ascending Testis
}

\author{
Stamatiou Konstantinos, Alevizos Alevizos, Mariolis Anargiros, Mihas Constantinos, \\ Halazonitis Athanase, Bovis Konstantinos, Eleftherios Michail, Sofras Fragiskos \\ Department of Urology (SK, BK, EM), Tzaneion General Hospital, Athens, Greece, Urban Health \\ Center of Vyronas (AA, MA, MC, HA), Athens, Greece, and Department of Urology (SF), School of \\ Medicine, University of Crete, Heraklion, Greece
}

\begin{abstract}
Objective: To prospectively determine the prevalence of testicular microlithiasis in symptomatic patients who were referred for scrotal ultrasound examination and to evaluate the possible association of testicular microlithiasis with testicular cancer and other conditions such as cryptorchidism or history of ascending testis.

Materials and Methods: 391 men who were referred to our institutions between July 2002 and May 2005 for any type of symptoms from the testicles, underwent physical and scrotal ultrasound examination. The presence of testicular microlithiasis, the number of lesions and the involvement of both testicles in relation to the symptoms as well as the coexistence of other lesions were studied.

Results: Eighteen (4.6\%) of 391 men enrolled into the study had testicular microlithiasis. Two out of the eighteen patients $(11 \%)$ had concomitant testicular cancer, which was confirmed by pathological evaluation of the orchidectomy specimen. One of the patients with testicular microlithiasis presented a rising in biochemical tumor markers (LDH, and HCG) and underwent orchidectomy one year later. Five of the remaining $373(1.3 \%)$ patients without microlithiasis were diagnosed with testicular cancer. Thirty six men reported having a history of ascending testis, but none of them was found with testicular cancer. Two cases of testicular torsion in a cryptorchid position had testicular microlithiasis, but the orchidectomy specimen (after surgery) was negative for testicular cancer. The correlation between testicular cancer and testicular microlithiasis found in our study was statistically significant $(\mathrm{p}<0.05)$.

Conclusion: There seems to be an association between testicular microlithiasis and testicular cancer.
\end{abstract}

Key words: testis; lithiasis; ultrasound; cryptorchidism; testicular neoplasms

Int Braz J Urol. 2006; 32: 434-9

\section{INTRODUCTION}

Testicular microlithiasis is a relatively uncommon condition characterized by calcifications within the testicular parenchyma visible through ultrasonographic imaging, which was firstly reported by Priebe \& Garret in the early 70's (1). Disseminated microcalcifications in various numbers, can be found inside the seminiferous tubules of the testicular parenchyma in less than $9 \%$ of symptomatic patients referred for scrotal ultrasonography (2-5), however, this incidence seems to increase with the 
use of high-frequency ultrasound devices (6). The clinical importance of entities such as testicular microlithiasis (in terms of symptoms and potential malignancy), is not well documented. Microcalcifications themselves are not malignant but they have been found in testes, in association with germ cell tumors in variable proportions. In the last few years, testicular microcalcifications have been considered an imaging marker of testicular cancer, with several authors recommending a serial scrotal ultrasound screening in patients with testicular microlithiasis, in order to detect possible testicular tumors in asymptomatic patients (7). Although some reports have indicated a prevalence of testicular microlithiasis in asymptomatic men referred for testicular ultrasound between $0.6 \%-0.7 \%$ (8-10), the actual prevalence of testicular microlithiasis in the general population is unknown. The aim of our study was to determine the prevalence of testicular microlithiasis in symptomatic patients who were referred for scrotal ultrasound examination and to evaluate the association of testicular microlithiasis with testicular cancer and other non-malignant conditions.

\section{MATERIALS AND METHODS}

Patients in this study included 468 men who were referred to our institution between July 2002 and May 2005 for any type of symptoms from the testicles (feeling of weight, pain, painful or painless inflammation, etc). All were aged between 15 and 76 years (median age 45.5 mean age 37 ) and all were from Athens and nearby suburbs. Men were eligible for enrollment if they never had a previous diagnosis of testis cancer, previous scrotal infection or history of testis surgery, with the exception of surgery cases regarding cryptorchidism. Of the 77 patients who were excluded from the study, 52 had a history of previous scrotal infection, while the remaining 15 had a history of testis surgery. All patients underwent physical and laboratory examination and were subjected to a scrotal ultrasound examination which was performed by 2 expert radiologists (HA, MC). Ultrasonographic criteria for the diagnosis of testicular microlithiasis were the presence of at least 5 pinpoint hyperechoic foci of size smaller than $3 \mathrm{~mm}$ in one field of view without posterior shadowing (11). The presence of testicular microlithiasis, the number of lesions and the involvement of both testicles in relation to the symptoms and the coexistence of other lesions were studied. Patients found with testicular microlithiasis were further investigated with biochemical tumor markers, chess $\mathrm{x}$ ray and if necessary with abdominal and pelvic computed tomography (CT) and placed in biannual follow-up with physical examination and scrotal ultrasound. All patients underwent the appropriate therapy. The association of pathologically confirmed testicular microlithiasis and testicular cancer were assessed with statistical analyses. A p value less than 0.05 was considered to indicate a statistically significant difference.

\section{RESULTS}

Of the 468 patients eligible, 391 fulfilled the inclusion criteria and were finally enrolled in the study. Eighteen (4.6\%) out of 391 men who enrolled in the study had testicular microlithiasis (Figure-1). According to the data obtained from the medical history of each participant, thirty six men reported having a history of ascending testis, while two of them were diagnosed in our institutions with torsioned testis in a cryptorchid abdominal position (probably ascending testis), in which the orchidectomy specimen (after surgery) was negative for testicular cancer. None of the three cases with testis surgery in early childhood for cryptorchidism had testicular microlithiasis. Testicular microlithiasis was the only finding in 10 patients, three patients had a concomitant varicocele, while both cases of torsioned cryptorchid testis had testicular microlithiasis in orchidectomy specimen. From 12 patients diagnosed with spermatic cord torsion only one had testicular microlithiasis in orchidectomy specimen. From the remaining patients 149 had epididymitis, 78 patients had varicocele, 24 patients had blunt trauma on left or right testis, 17 had hernia, 14 spermatocele, 13 had an urinary stone in the lower third of the left or right ureter, and 11 patients had periorchitis clinically 


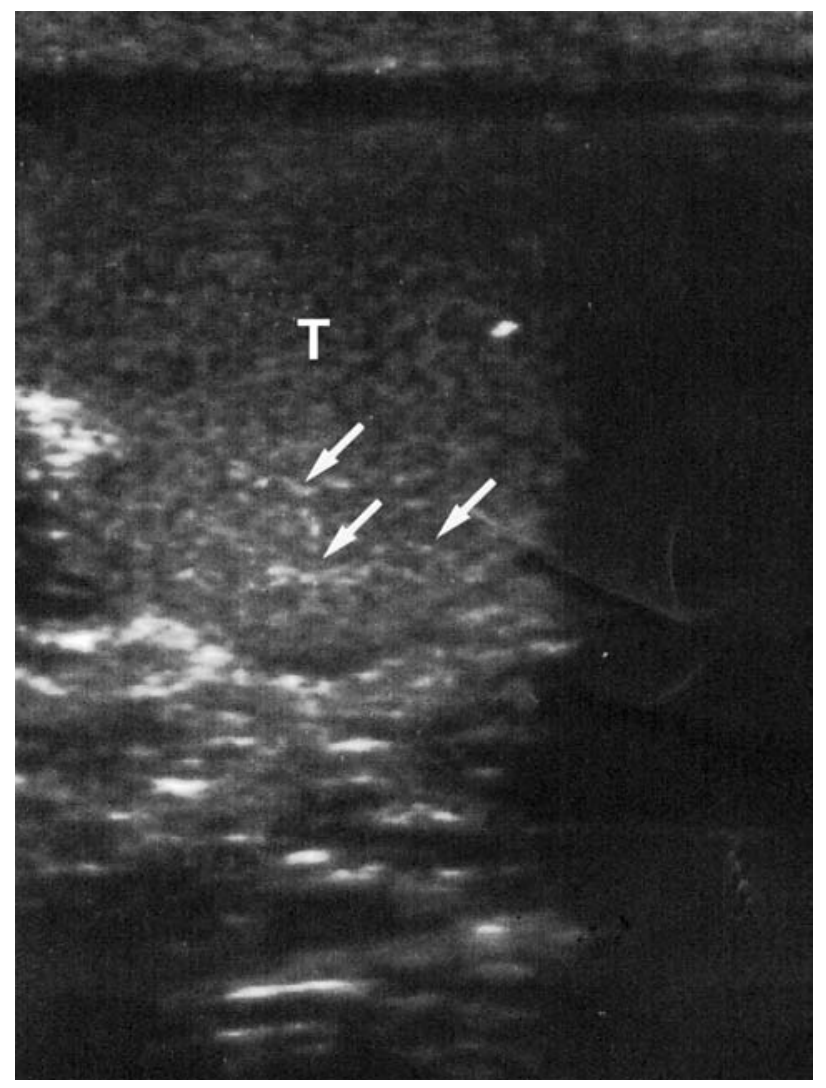

Figure 1 - Ultrasonographic image of a patient with bilateral testicular microlithiasis.

diagnosed and confirmed with both laboratory and echographic examination. None of them had microcalcifications (in terms of testicular microlithiasis) in his scrotum ultrasound. Despite the extensive investigation, no evident cause for the symptoms was obtained for 51 men, and they have been dismissed from the urologic department without a urology-related diagnosis. Seven patients had testicular cancer diagnosed with both biochemical tumor markers and echographic examination and also confirmed by pathologic examination. All cases of testicular cancer were germ cell tumors. Two of them had coexistent testicular microliths (11.1\%) in the orchidectomy specimen, evidenced in the pathologic examination as hematoxylin bodies. Interestingly the number of calcifications in these cases was relatively higher than that obtained from normal testes. Only five of the 373 (1.3\%) patients without microlithiasis were found with an overt testicular cancer. One year after the initiation of follow up, in one of the patients with testicular microlithiasis a rise in testicular tumor markers (LDH, and HCG) was encountered and orchidectomy was performed.

Despite the low number of cases, the correlation between testicular cancer and testicular microlithiasis found in our study was confirmed with the Fisher exact statistic, at a significance level less than 0.05 . The most commonly reported symptoms related to the presence of testicular microlithiasis alone were pain and/or discomfort reported by all patients with testicular microlithiasis alone. Additional symptoms such as painful or painless inflation, fever and scrotal tenderness are probably connected to coexistent conditions.

\section{COMMENTS}

Since the first detailed description of this ultrasonographic entity (12) the biological meaning remains unclear. Whether and if testicular microlithiasis is present before the development of testicular germ cell tumors and the time needed to develop testicular cancer is not well known thus the prognostic value of this entity as a precancerous lesion for testicular cancer remains controversial (13). Although several authors suggested that testicular microlithiasis should be considered a premalignant condition, wide variation in the reported incidence of testicular microlithiasis in men with concomitant germ cell malignancy (6-75\%) indicates that probably it is not the case $(1,14-16)$. Other studies have showed that bilateral microlithiasis has been associated to the pre-invasive stage of germ cells testicular cancer more than unilateral $(17,18)$, but this finding could be the effect of bias since testicular microlithiasis usually occurs bilaterally $(2,19)$. On the other hand, testicular microlithiasis is found more often in men with concomitant benign testicular conditions [cryptorchidism, testicular dysgenesis, male infertility, testicular torsion and atrophy, Klinefelter's syndrome, hypogonadism, male pseudohermaphroditism, varicocele, and epididymal 
cysts (20-22)] proposing that the microcalcifications themselves are not malignant. Indeed, ultrasonic microliths may be visible for many reasons, for example histological microliths, hyalinased tubules, carcinoma in situ, or for unknown reasons (23) and thus are heterogeneous. Pathologically, 2 types of calcifications have been described (24): hematoxylin bodies consisting of amorphous calcific debris and laminated calcifications consisting of cellular debris and glucoprotein accumulation.: while hematoxylin bodies are not seen in the absence of germ cell neoplasia and are considered specific for germ cell tumors, laminated calcifications which are even more common in germ cell tumors also occur in otherwise normal testis. The pathogenesis of laminated microcalcifications is probably due to dysgenesis of the testis, with slough of degenerated cells inside an obstructed seminiferous tubule and failure of the Sertoli cells to phagocytize the debris. Secondarily, calcification occurs. This may explain why microlithiasis is found to accompany both germ cell tumors and non-malignant conditions connected to infertility. Ultrasonic microliths coexistent with germ cell tumors were histologically found to be hematoxylin bodies. In our study group, the incidence of testicular microlithiasis $(4.6 \%)$ and the percentage of patients with testicular cancer among testicular microlithiasis patients $(11 \%)$, were in accordance with most of the aforementioned studies (0.6-9\% and 6-75\% respectively). In addition, our finding of rapid onset of testis cancer (within 15 months) in a patient with known testicular microlithiasis, is in agreement with several authors (19), although in other studies testicular cancer has been developed even seven years after the primary diagnosis of testicular microlithiasis (25). Furthermore, since in our study testicular microlithiasis was not associated with any individual symptom or any non-malignant condition from the testis, we believe that the detection of testicular microlithiasis can probably be attributed to chance. In conclusion, we hope that our study adds more evidence in the association between testicular microlithiasis and testicular cancer. Moreover, although it is not known whether and when a patient with testicular microlithiasis would ever develop testicular cancer, we totally agree with the current recommen- dations and similarly suggest that patients with evidence of testicular microlithiasis should be screened for testicular cancer in a regular basis.

\section{CONFLICT OF INTEREST}

None declared.

\section{REFERENCES}

1. Priebe CJ Jr, Garret R: Testicular calcification in a 4year-old boy. Pediatrics. 1970; 46: 785-8.

2. Parra BL, Venable DD, Gonzalez E, Eastham JA: Testicular microlithiasis as a predictor of intratubular germ cell neoplasia. Urology. 1996; 48: 797-9.

3. Bach AM, Hann LE, Shi W, Giess CS, Yoo HH, Sheinfeld J, et al.: Is there an increased incidence of contralateral testicular cancer in patients with intratesticular microlithiasis? AJR Am J Roentgenol. 2003; 180: 497-500.

4. Ringdahl E, Claybrook K, Teague JL, Northrup M: Testicular microlithiasis and its relation to testicular cancer on ultrasound findings of symptomatic men. $\mathrm{J}$ Urol. 2004; 172: 1904-6.

5. Hobarth K, Susani M, Szabo N, Kratzik C: Incidence of testicular microlithiasis. Urology. 1992; 40: 464-7.

6. Pierik FH, Dohle GR, van Muiswinkel JM, Vreeburg JT, Weber RF: Is routine scrotal ultrasound advantageous in infertile men? J Urol. 1999; 162: 161820.

7. Winter TC 3rd, Zunkel DE, Mack LA: Testicular carcinoma in a patient with previously demonstrated testicular microlithiasis. J Urol. 1996; 155: 648.

8. Peterson AC, Bauman JM, Light DE, McMann LP, Costabile RA: The prevalence of testicular microlithiasis in an asymptomatic population of men 18 to 35 years old. J Urol. 2001; 166: 2061-4.

9. Cast JE, Nelson WM, Early AS, Biyani S, Cooksey G, Warnock NG, et al.: Testicular microlithiasis: prevalence and tumor risk in a population referred for scrotal sonography. AJR Am J Roentgenol. 2000; 175: 1703-6.

10. Pourbagher MA, Kilinc F, Guvel S, Pourbagher A, Egilmez T, Ozkardes H: Follow-up of testicular microlithiasis for subsequent testicular cancer development. Urol Int. 2005; 74: 108-12. 
11. Bach AM, Hann LE, Hadar O, Shi W, Yoo HH, Giess CS, et al.: Testicular microlithiasis: what is its association with testicular cancer? Radiology. 2001; 220: 70-5.

12. Doherty FJ, Mullins TL, Sant GR, Drinkwater MA, Ucci AA Jr: Testicular microlithiasis. A unique sonographic appearance. J Ultrasound Med. 1987; 6: 389-92.

13. Miller FN, Sidhu PS: Does testicular microlithiasis matter? A review. Clin Radiol. 2002; 57: 883-90.

14. Derogee M, Bevers RF, Prins HJ, Jonges TG, Elbers FH, Boon TA: Testicular microlithiasis, a premalignant condition: prevalence, histopathologic findings, and relation to testicular tumor. Urology. 2001; 57: 1133-7.

15. Giwercman A, Muller J, Skakkebaek NE: Prevalence of carcinoma in situ and other histopathological abnormalities in testes from 399 men who died suddenly and unexpectedly. J Urol. 1991; 145: 77-80.

16. Patel MD, Olcott EW, Kerschmann RL, Callen PW, Gooding GA: Sonographically detected testicular microlithiasis and testicular carcinoma. J Clin Ultrasound. 1993; 21: 447-52.

17. de Gouveia Brazao CA, Pierik FH, Oosterhuis JW, Dohle GR, Looijenga LH, Weber RF: Bilateral testicular microlithiasis predicts the presence of the precursor of testicular germ cell tumors in subfertile men. J Urol. 2004; 171: 158-60.

18. Roberts IS, Loughran CF: Case report: the ultrasound appearances of testicular microlithiasis ('snow storm' testis): a case complicated by testicular seminoma. Clin Radiol. 1993; 47: 65-7.

19. Frush DP, Kliewer MA, Madden JF: Testicular microlithiasis and subsequent development of metastatic germ cell tumor. AJR Am J Roentgenol. 1996; 167: 889-90.

20. Vegni-Talluri M, Bigliardi E, Vanni MG, Tota G: Testicular microliths: their origin and structure. J Urol. 1980; 124: 105-7.

21. von Eckardstein S, Tsakmakidis G, Kamischke A, Rolf C, Nieschlag E: Sonographic testicular microlithiasis as an indicator of premalignant conditions in normal and infertile men. J Androl. 2001; 22: 818-24.

22. Thomas K, Wood SJ, Thompson AJ, Pilling D, LewisJones DI: The incidence and significance of testicular microlithiasis in a subfertile population. Br J Radiol. 2000; 73: 494-7.

23. Holm M, Hoei-Hansen CE, Rajpert-De Meyts E, Skakkebaek NE: Increased risk of carcinoma in situ in patients with testicular germ cell cancer with ultrasonic microlithiasis in the contralateral testicle. J Urol. 2003; 170: 1163-7.

24. Renshaw AA: Testicular calcifications: incidence, histology and proposed pathological criteria for testicular microlithiasis. J Urol. 1998; 160: 1625-8.

25. Furness PD 3rd, Husmann DA, Brock JW 3rd, Steinhardt GF, Bukowski TP, Freedman AL, et al.: Multi-institutional study of testicular microlithiasis in childhood: a benign or premalignant condition? J Urol. 1998; 160: 1151-4.

Correspondence address:

Dr. Stamatiou Konstantinos

Salepoula 2, 18536

Piraeus, Greece

Fax: + $30210429-6987$

E-mail: alvise@tellas.gr 


\section{EDITORIAL COMMENT}

The answer to the question "Should testicular microlithiasis be regarded as a precursor of testicular cancer?" is still unclear. Actually, the most important information obtained from this prospective study is the onset of testis cancer during follow-up of a patient wit testicular microlithiasis. In the literature so far, there are only 13 reports related to 15 patients with testicular microlithiasis who subsequently had testicular cancer. Three of these 13 reports were retrospective studies. Derogee et al. followed 31 patients with testicular microlithiasis from 16 to 105 months and a combined teratoma and seminoma developed in one patient. Otite et al. followed 38 patients with testicular microlithiasis and two of these patients under surveillance developed a testicular malignancy in which one patient had an atrophic left testis and left testicular tumor developed. von Eckardstein fol- lowed 14 men with testicular microlithiasis for 24 to 60 months and testicular tumors developed in two of them. Review of the literature reveals that 4 of the 15 patients had a history of contralateral testicular tumor. Three patients had also an infertility problem. The remaining 7 patients with testicular microlithiasis had no associated abnormality that could be related to tumor development.

Regarding the higher prevalence of testicular tumors, this number seems very low in order to accept testicular microlithiasis as a premalignant disease. However, until the relationship between testicular microlithiasis and testicular tumors is clearly defined, if they will ever be, annual follow-up with scrotal ultrasonography and physical examination seems still appropriate and will aid to establish more evidence based guidelines.

Dr. Tulga M. Egilmez

Dept of Urology, Baskent Univ School Medicine Adana Teaching \& Medical Research Center Adana, Turkey

E-mail: tulgaegilmez@yahoo.com 